\title{
Identifying the hub genes for Duchenne muscular dystrophy and Becker muscular dystrophy by weighted correlation network analysis
}

Junjie Wang ${ }^{1}$, Qin Fan ${ }^{1}$, Tengbo Yu ${ }^{1,2^{*}}$ and Yingze Zhang ${ }^{1,2,3^{*}}$

\begin{abstract}
Background: The goal of this study is to identify the hub genes for Duchenne muscular dystrophy (DMD) and Becker muscular dystrophy (BMD) via weighted correlation network analysis (WGCNA).

Methods: The gene expression profile of vastus lateralis biopsy samples obtained in 17 patients with DMD, 11 patients with BMD and 6 healthy individuals was downloaded from the Gene Expression Omnibus (GEO) database (GSE109178). After obtaining different expressed genes (DEGs) via GEO2R, WGCNA was conducted using R package, modules and genes that highly associated with $\mathrm{DMD}, \mathrm{BMD}$, and their age or pathology were screened. Gene Ontology (GO) and Kyoto Encyclopaedia of Genes and Genomes (KEGG) enrichment analysis and protein-protein interaction (PPI) network analysis were also conducted. Hub genes and highly correlated clustered genes were identified using Search Tool for the Retrieval of Interacting Genes (STRING) and Cystoscape software.
\end{abstract}

Results: One thousand four hundred seventy DEGs were identified between DMD and control, with 1281 upregulated and 189 downregulated DEGs. Four hundred and twenty DEGs were found between BMD and control, with 157 upregulated and 263 upregulated DEGs. Fourteen modules with different colors were identified for DMD vs control, and 7 modules with different colors were identified for BMD vs control. Ten hub genes were summarized for DMD and BMD respectively, 5 hub genes were summarized for BMD age, 5 and 3 highly correlated clustered genes were summarized for DMD age and BMD pathology, respectively. In addition, $20 \mathrm{GO}$ enrichments were found to be involved in DMD, $3 \mathrm{GO}$ enrichments were found to be involved in BMD, $3 \mathrm{GO}$ enrichments were found to be involved in BMD age.

Conclusion: In DMD, several hub genes were identified: C3AR1, TLR7, IRF8, FYB and CD33(immune and inflammation associated genes), TYROBP, PLEK, AIF1 (actin reorganization associated genes), LAPTM5 and NT5E(cell death and arterial calcification associated genes, respectively). In BMD, a number of hub genes were identified: LOX, ELN, PLEK, IKZF1, CTSK, THBS2, ADAMTS2, COL5A1 (extracellular matrix associated genes), BCL2L1 and CDK2(cell cycle associated genes).

Keywords: Duchenne muscular dystrophy, Becker muscular dystrophy, Gene expression omnibus, Weighted correlation network analysis

*Correspondence: ytb1970@163.com; suryzz1953@126.com

${ }^{2}$ Orthopaedic Center, The Affiliated Hospital of Qingdao University, No.

16, Jiangsu Road, Qingdao, Shandong Province 266000, China

Full list of author information is available at the end of the article

\section{Background}

Duchenne muscular dystrophy (DMD) and Becker muscular dystrophy (BMD) are X-linked recessive diseases, the major genetic alterations are mutations in dystrophin 
gene [1]. Dystrophin is a part of the dystrophin-glycoprotein complex (DGC), which provides structural stability at the sarcolemma during muscle contraction by linking the internal cell cytoskeleton and external extracellular matrix [2]. Mutations in dystrophin gene can lead to reduction, abnormal or absence of DGC, as a result, degeneration in neuromuscular function occurs [3]. The main symptom of DMD is progressive muscle weakness. DMD patients usually present symptom by age 3 to 5 years, and they successively lose lower and upper limbs function before their adulthood, the most common causes of death for DMD include respiratory and cardiac failure by their 20 to 30 years. It is estimated that nearly half number of patients fail to live to their 20 years old [4]. Compared with DMD, the symptoms of BMD are similar but relatively milder [5]. In addition, the onset, progression, presentation and severity of BMD seem to be more heterogeneous among patients. For example, time point for loss of ambulation ranging from 16years old to 70 years old [6]. According to worldwide history of newborn screening, the incidence for DMD ranges from 1 in 4589 to 6291 livebirths, and most of them are males, rating as the most common form of muscular dystrophy in children [7]. While according to a meta-analysis, the incidence of BMD is about 2.21 in 100,000 livebirths and males are also the most affected [8].

Currently, the major management for DMD and BMD remains symptomatic treatment such as corticosteroids therapy, wheelchair, ventilation, cough assists and treatment of cardiomyopathy [9]. Although these care can extend life expectancy to some extent [10], DMD and BMD patients still need more effective therapy to treat diseases in order to improve their life quality. Since DMD and BMD are genetic disorders, gene-targeted therapy seems to be a feasible method. However, it is reported that genetic therapy is not usually helpful for a patient who has already lost a substantial part of his muscle tissue and function [11]. Therefore, it is necessary to explore hub genes in order to deeply understand genetic etiology and provide new insights into the early diagnosis and treatment that can be targeted in the pharmaceutical strategy. To the best of our knowledge, although a recent article has identified hub genes for DMD and BMD via weighted correlation network analysis (WGCNA) [12], it is still necessary to identify hub genes for DMD and BMD via WGCNA using differentially expressed genes (DEGs).

In this article, we aim to explore the hub genes for DMD and BMD via WGCNA using DEGs.

\section{Materials and methods Data collection}

The gene expression profiles of patients with DMD and $\mathrm{BMD}$, and healthy control were downloaded from the Gene Expression Omnibus (GEO) database. The GSE109178 microarray dataset was used for bioinformatic analysis. GSE109178 (GPL570, Affymetrix Human Genome U133 Plus 2.0 Array) used vastus lateralis biopsy samples obtained from 17 patients with DMD, 11 patients with BMD and 6 healthy individuals.

\section{Identifying DEGs}

GEO2R is an online tool for identifying differentially expressed molecules across various experimental conditions, and it was utilized to identify DEGs between DMD vs control and BMD vs control DEGs were defined from analysis of the microarray data with adjusted $P$ value $<0.05$ and $\mid \log 2$ fold change (FC) $\mid>1.5$ as cutoffs. The normalization of datasets and limma precision weight analysis were also conducted with GEO2R.

\section{WGCNA}

After obtaining DEGs via GEO2R, WGCNA was conducted using an $\mathrm{R}$ package. The adjacency matrix was converted into a topological overlap matrix (TOM). A soft-thresholding power was set, and DEGs were divided into different modules. Modules and clustered genes that were highly associated with DMD, BMD or their age and pathology (such as mild, moderate or severe symptom) were screened ( $\mid$ correlativity $\mid>0.5)$.

\section{Gene ontology (GO) and Kyoto encyclopedia of genes and genomes (KEGG) pathway enrichment analyses}

$\mathrm{GO}$ is a major bioinformatics tool for annotating genes and analysing their biological processes. KEGG is a database resource for understanding the high-level functions and biological systems of large-scale molecular data generated by high-throughput experimental technologies. To deeply explore the biological functions of highly correlated clustered DEGs between DMD vs control and BMD vs control, WebGestalt (http://www.webgestalt.org/) version 2019, a functional enrichment analysis web tool, was used to conduct GO and KEGG pathway enrichment analyses. A false discovery rate (FDR) $\leq 0.05$ was considered statistically significant.

\section{Protein-protein interaction (PPI) network construction and hub genes identification}

Search Tool for the Retrieval of Interacting Genes (STRING; http://string-db.org) (version 11.0), a webbased tool that analyses the functional interactions among proteins, was used to build a PPI network of the highly correlated clustered DEGs. Cytoscape is an open source software platform for visualizing complex networks and combining them with any type of attribute data. The information in STRING was imported into Cytoscape (version 3.7.1), and the PPI network of highly 
correlated clustered DEGs was established. The top 10 hub genes were identified according to 12 algorithms.

\section{Results}

Normalization of dataset

Figure $1 \mathrm{a}$ and $\mathrm{b}$ shows the results of the normalization of the dataset, which indicate a relatively high consistency between groups.

\section{Identification of DEGs}

After deleting pseudogene, 1470 DEGs between DMD and control were identified, with 1281 upregulated genes and 189 downregulated genes for DMD. Four hundred and twenty DEGs between BMD and control were found, with 157 upregulated genes and 263 downregulated genes for BMD (Fig. 2a and b).

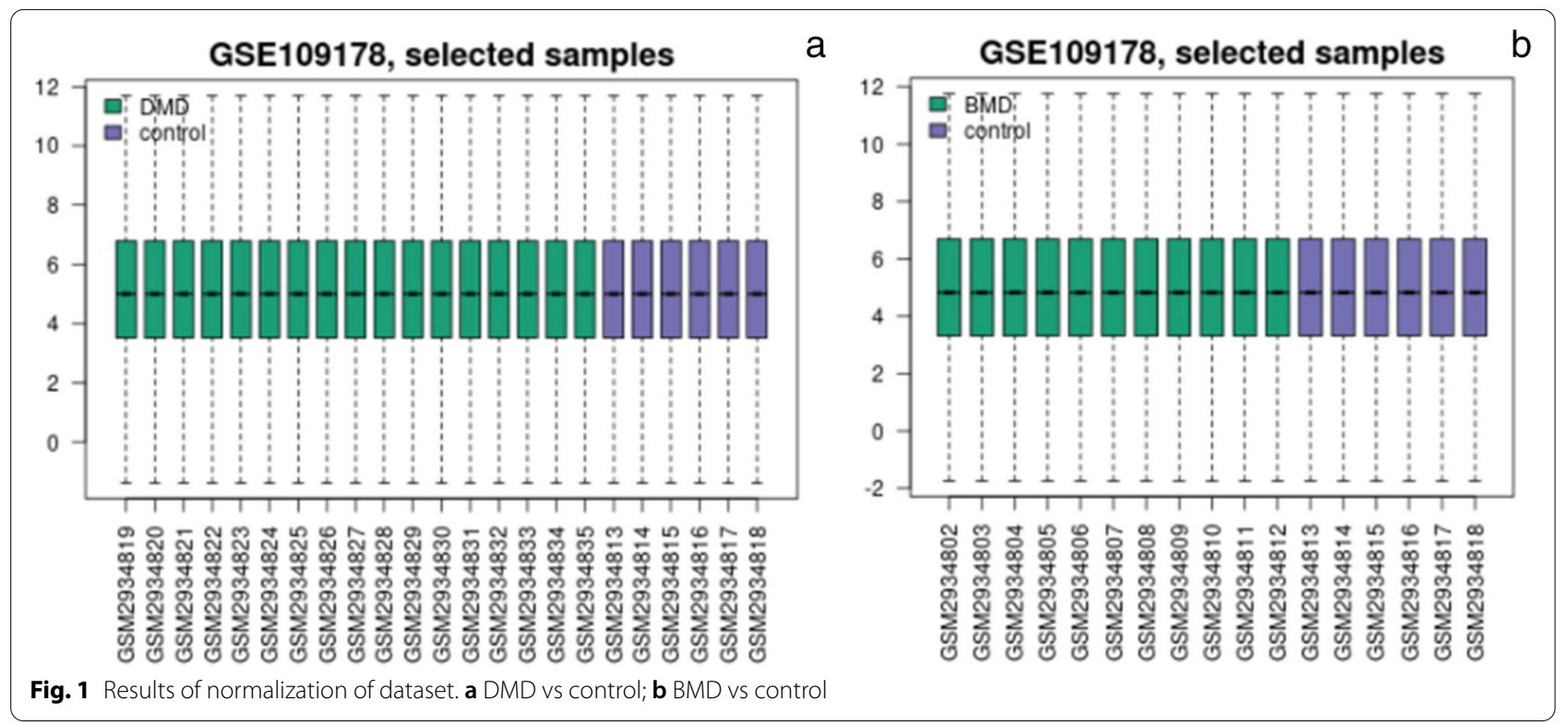

a GSE109178: Asynchronous remodeling is a driver of failed regeneration... DMD vs control, Padj $<0.05$

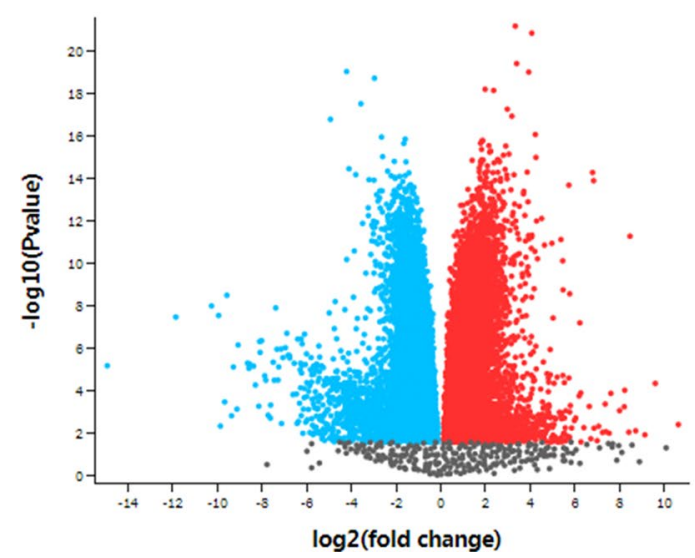

Fig. 2 Volcano plots of all DEGs. a DMD vs control; b BMD vs control

\section{Volcano plot \\ GSE109178: Asynchronous remodeling is a driver of failed regeneration... $B M D$ vs control, $P a d j<0.05$}

b

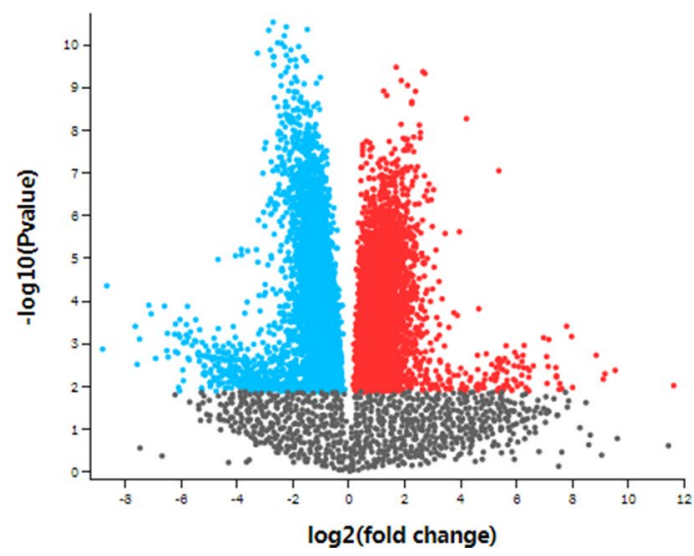




\section{WGCNA}

The DEGs were then assessed with WGCNA. Figure 3a and $b$ shows that the soft-thresholding power was determined to be $\beta=18$, at which point the curve first achieved $\mathrm{R}^{\wedge} 2=0.82$ for $\mathrm{DMD}$ vs control, and $\beta=8$ at which point the curve first achieved $\mathrm{R}^{\wedge} 2=0.81$ for $\mathrm{BMD}$ vs control. Subsequently, a TOM-based dissimilarity measure was applied, 14 modules with different colours were identified for DMD vs control, and 7 modules with different colours were identified for BMD vs control, as presented in the dendrogram plots (Fig. 4a and b). In addition, correlation plots between the module colours or genes and clinical traits was constructed (Fig. $5 \mathrm{a}$ and b).

\section{GO and KEGG pathway enrichment analyses}

The results of GO enrichment analysis for the highly correlated clustered DEGs are shown in Fig. 6a and b. The specific enrichment results showed that for DMD, genes were enriched in immune response, myeloid leukocyte activation, regulated exocytosis, cell activation, neutrophil degranulation, neutrophil activation involved in immune response, defense response, neutrophil activation, neutrophil mediated immunity, and granulocyte activation in the biological process (BP) category, secretory vesicle, secretory granule, lysosome, lytic vacuole, vacuolar part, cytoplasmic vesicle part, vacuole, ruffle, vacuolar lumen, and whole membrane in cellular component $(\mathrm{CC})$ category. For BMD, genes were enriched in extracellular matrix and collagen-containing extracellular matrix in CC category, extracellular matrix structural constituent in molecular function (MF) category. For BMD age, genes were enriched in extracellular matrix and collagen-containing extracellular matrix in CC, extracellular matrix structural constituent in MF category. FDR was more than 0.05 in all KEGG pathway enrichment items for DMD, BMD, age and pathology.

\section{PPI network and hub genes}

The PPI network of the highly correlated clustered DEGs was constructed using STRING and then imported into Cytoscape. Using the 12 algorithms in the CytoHubba plugin, hub genes or highly correlated genes for DMD, BMD, age and pathology were summarized (Table 1). The GO and KEGG pathway enrichment analysis that hub genes were enriched were summarized in Table 2.

\section{Discussion}

The process of losing muscle function in DMD and BMD patients starts from a very early age and is irreversible, therefore, the genetic intervention for DMD and BMD should be as early as possible, since genetic therapy is unable to restore muscle tissue that has already lost function. The current study employed WGCNA to identify highly correlated hub genes in samples of vastus lateralis from patients with DMD and BMD, and healthy control. As a result, 10 hub genes were summarized for DMD and BMD respectively, 5 hub genes were summarized for BMD age, 5 and 3 highly correlated clustered genes were summarized for in DMD age and BMD pathology, respectively. In addition, $20 \mathrm{GO}$ enrichment terms were found to be involved in DMD, $3 \mathrm{GO}$ enrichment terms were found to be involved in BMD, $3 \mathrm{GO}$ enrichment terms were found to be involved in BMD age.

Ten hub genes were identified for DMD and they are all positively correlated with DMD, among them, 5 were from turquoise module, 3 were from yellow module, 1 was from brown module and the last was from tan module. The five genes from turquoise module were C3AR1(encodes complement component 3a receptor 1), TLR7(encodes toll like receptor 7), IRF8(encodes interferon regulatory factor 8), FYB (encodes FYN binding protein) and CD33(encodes CD33 molecule). C3 molecule is a biomarker for muscle fiber diseases $[13,14]$. In addition, it has been demonstrated that C3 gene knockout can relieve muscle pathology in dysferlin-deficient mice [15]. Moreover, scientists have found that histone deacetylase inhibitors, which can attenuate DMD pathology, lowers C3 molecule level in DMD mice [16]. These may suggest the importance of C3AR1 protein and its gene upregulation in DMD. It has been observed that the expression of TLR7 gene increases in DMD mice, the upregulating TLR7 gene expression can induce inflammatory signaling pathway. Moreover, treating DMD mice with TLR7 molecule antagonist can clearly relieve skeletal muscle inflammation and improve muscle force [17]. This indicated the role of TLR7 gene as a potential therapeutic target for DMD. IRF8 protein is a crucial modulator of inflammation in immune cells [18]. FYN protein is a member of Src family kinase, it is also involved in inflammatory signaling pathway $[19,20]$. CD33 molecule is a myeloid antigen and play an essential role in the inflammation [21, 22]. Genes from turquoise module mainly participate in immune and inflammation, this suggested immune and inflammation play an important role in DMD, which is consistent with previous studies $[23,24]$. Three hub genes for DMD were from yellow module, included PLEK (encodes pleckstrin), TYROBP (encodes TYRO protein tyrosine kinase binding protein) and AIF1(encodes allograft inflammatory factor 1). Pleckstrin is thought to be involved in actin rearrangement [25], in addition, it is associated with platelets adhesion to collagen [26]. TYROBP protein is a part of inflammation signaling pathway that is associated with actin cytoskeleton reorganization [27]. AIF-1 protein is an actin binding protein and may related to actin rearrangement [28, 29]. The hub genes from yellow module are all associated with actin reorganization, this suggested that actin 


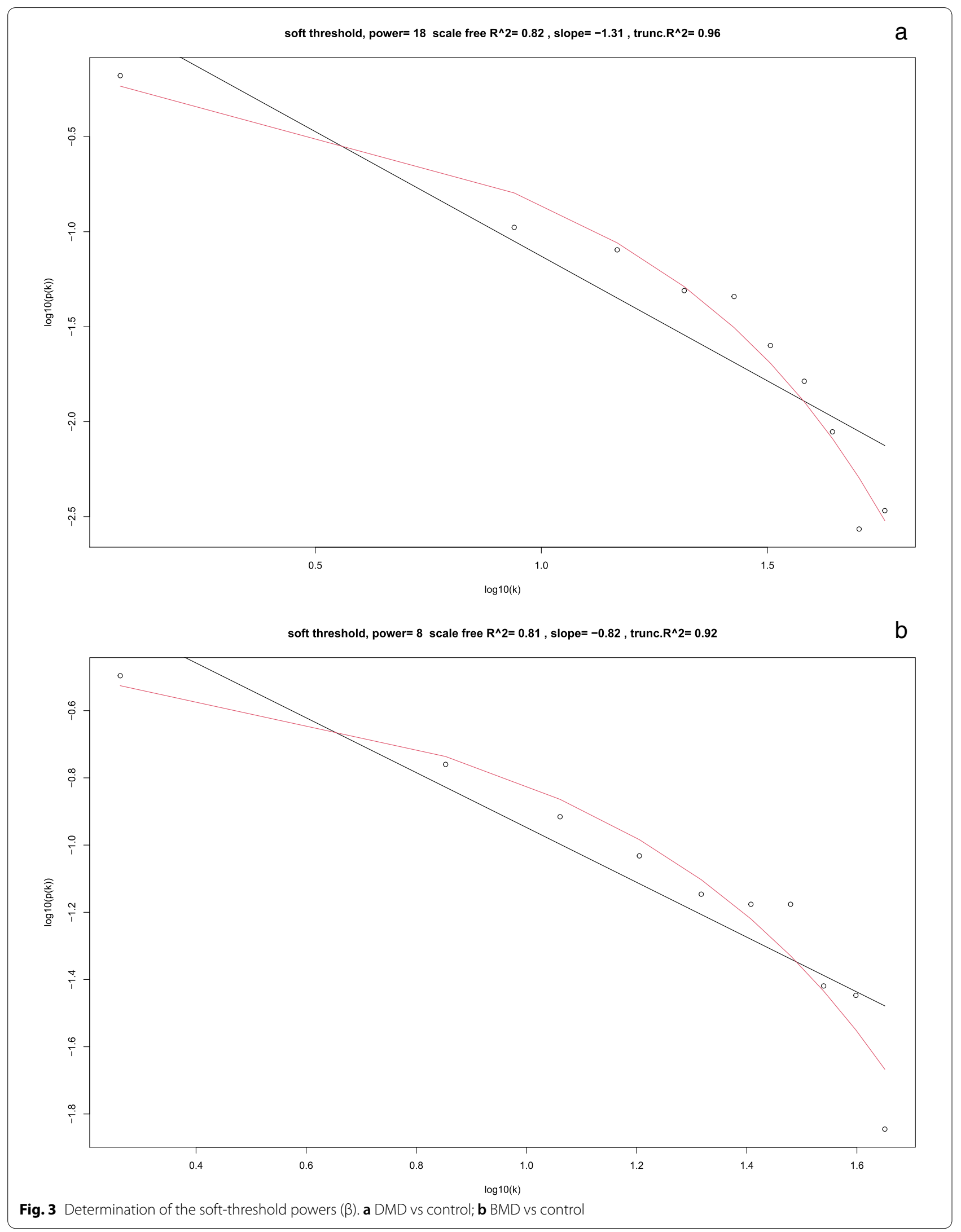



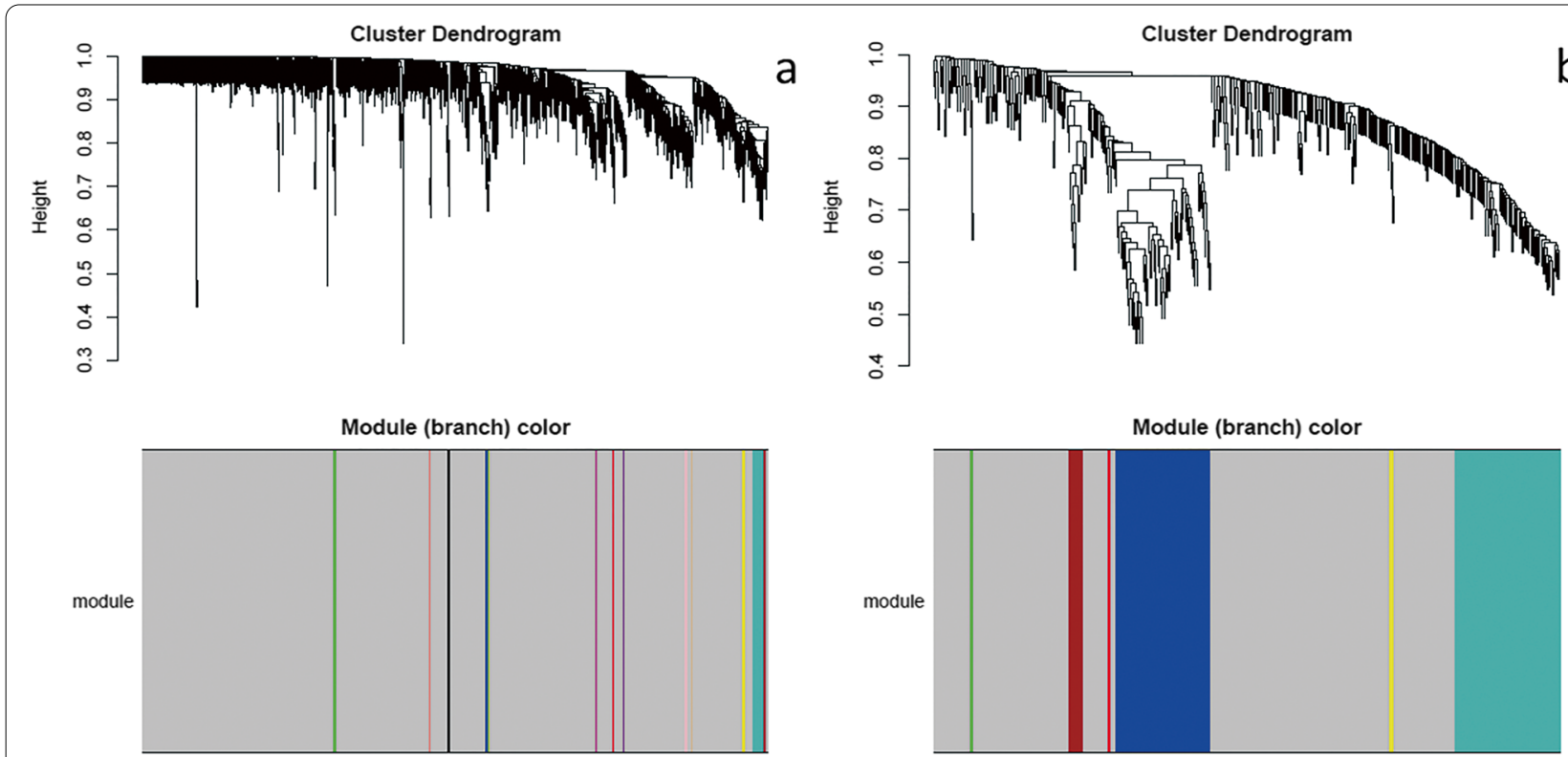

Fig. 4 The clustering dendrogram of DEGs, non-clustering DEGs were shown in grey. a DMD vs control; b BMD vs control

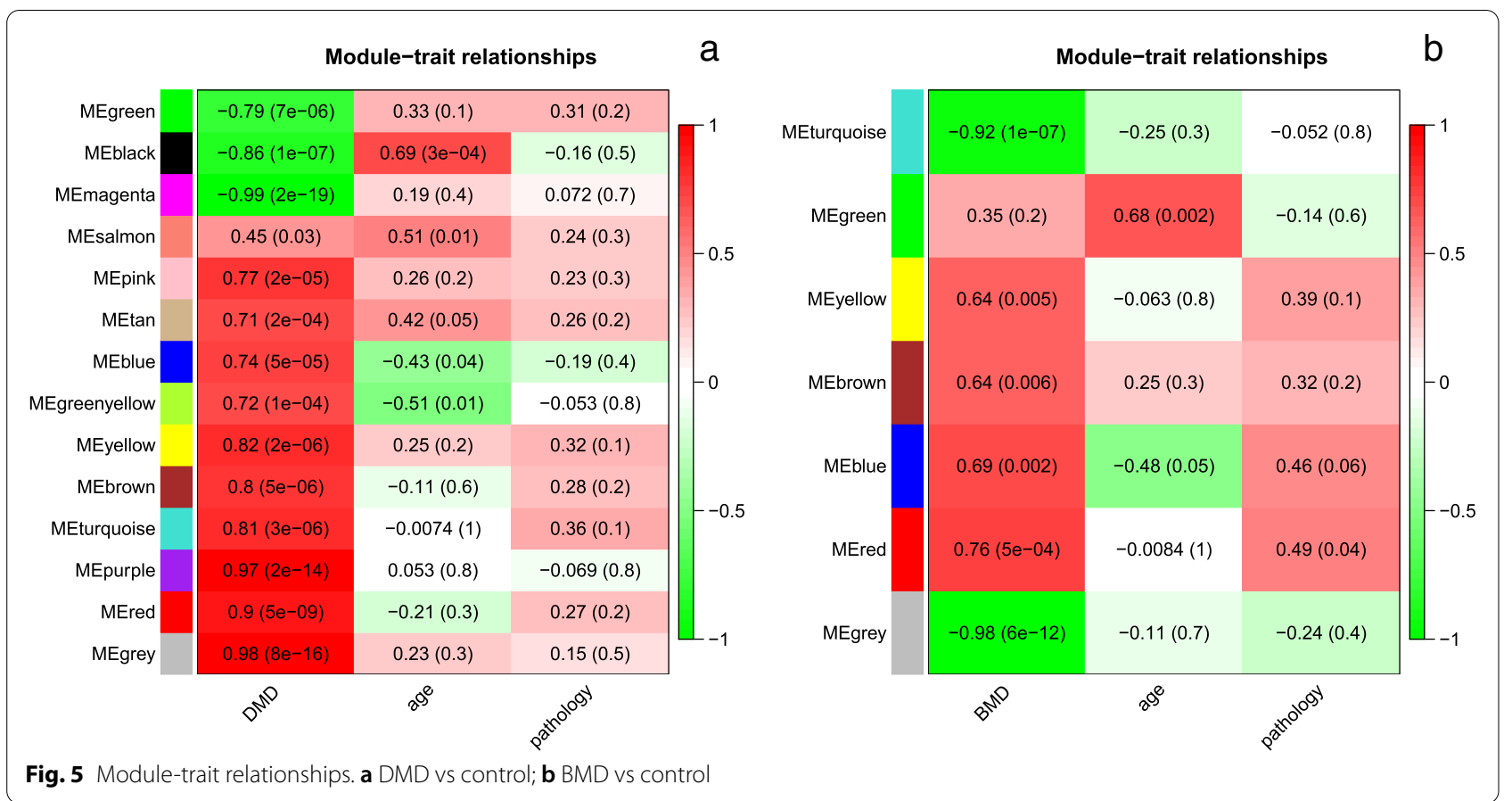

reorganization may play a vital role in DMD, this result is similar to a previous study [30]. LAPTM5(encodes lysosomal protein transmembrane 5) was from brown module, NT5E(encodes 5'-nucleotidase ecto) was from tan module, and they were both hub genes for DMD. Scientists have demonstrated that LAPTM5 gene is closely related to programmed cell death [31]. NT5E gene is mainly expressed in smooth muscle cells [32], its encoding protein can convert adenosine $5^{\prime}$-monophosphate to adenosine and is associated with arterial calcification [33]. It has been observed that arterial stiffness increases in DMD patients [34].

Five genes correlated with DMD age were identified, two of them were from black module, others were 

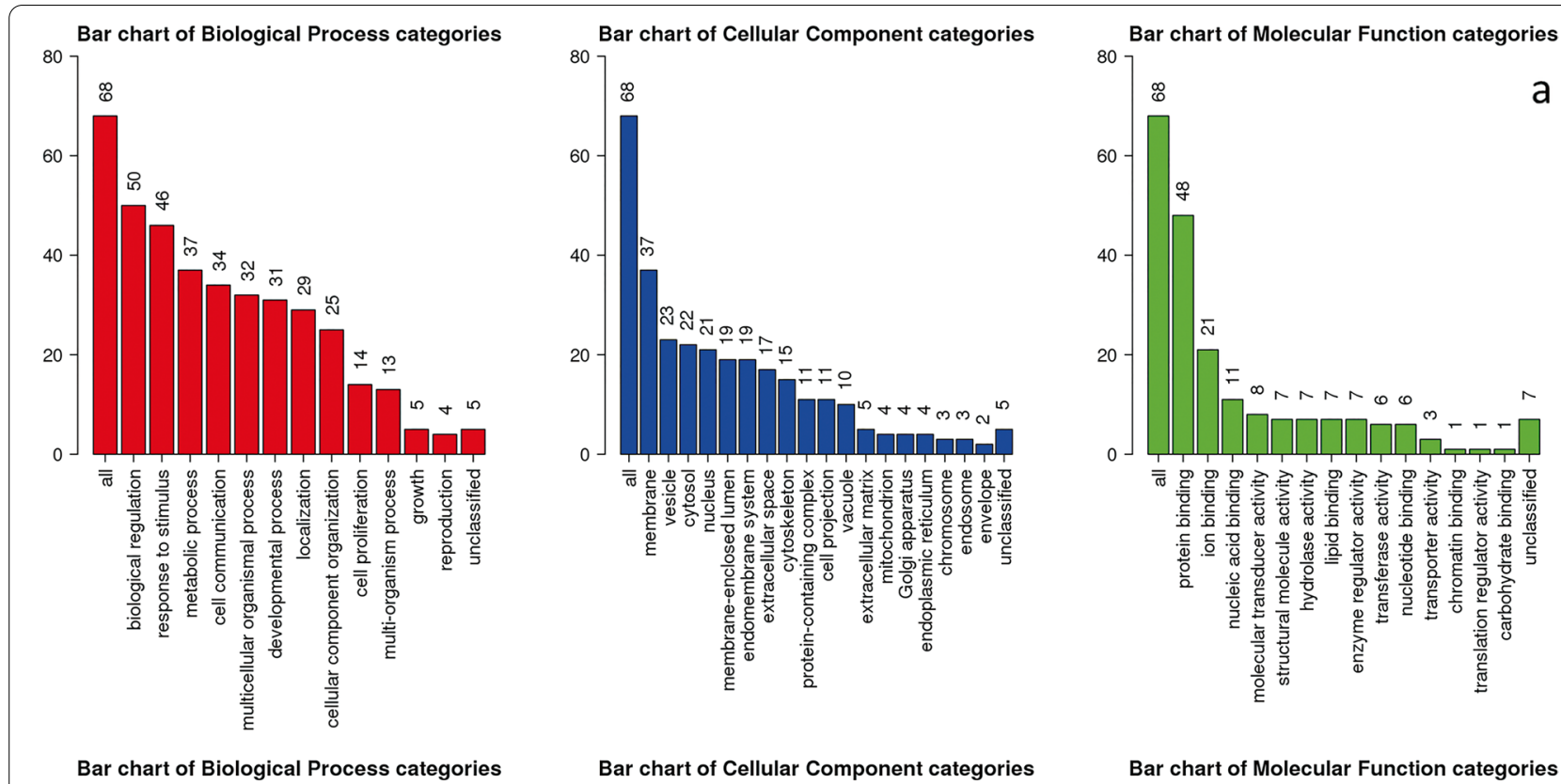

Bar chart of Molecular Function categories
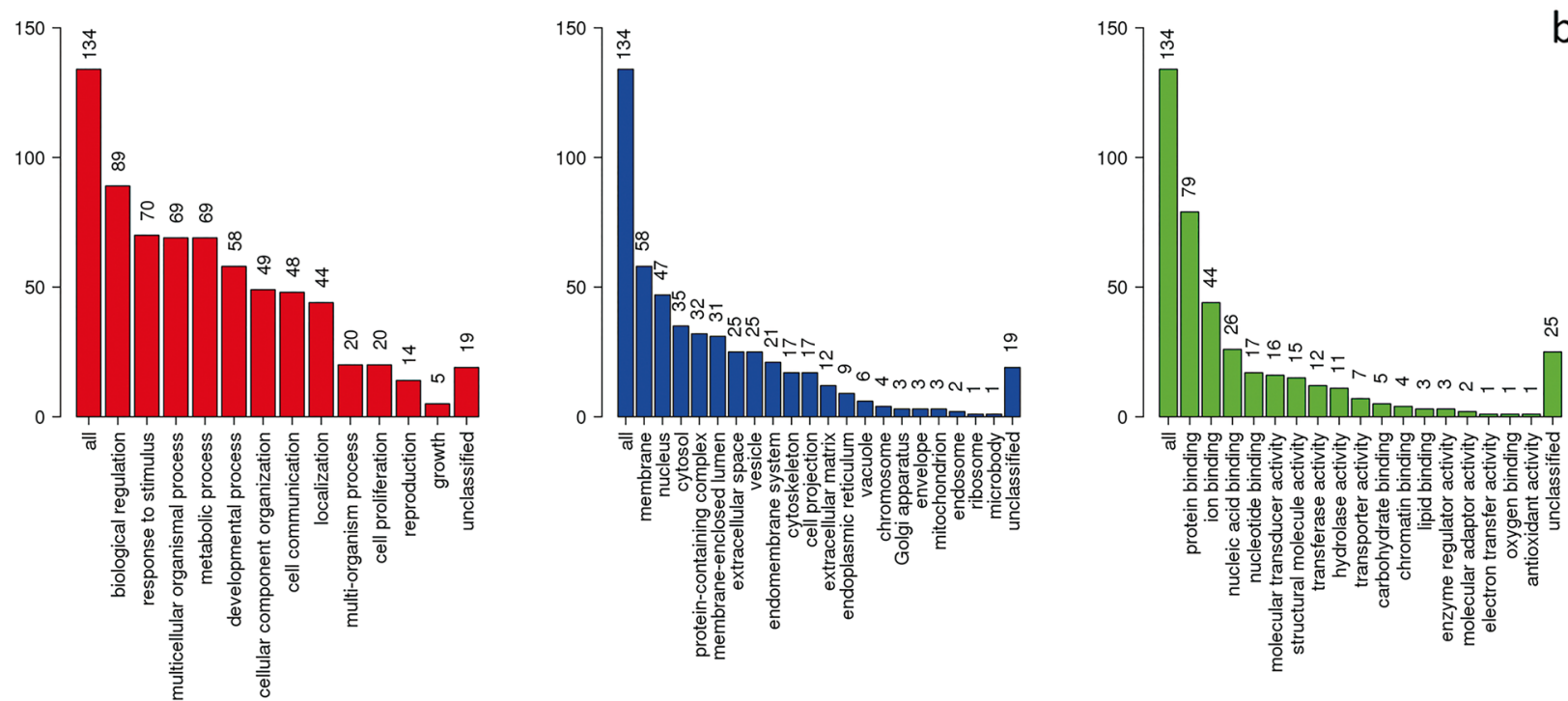

Fig. 6 GO enrichment analysis of the highly correlated clustered DEGs. a DMD vs control; b BMD vs control

from blue, salmon and greenyellow module, respectively. KRT31(encodes keratin 31), KRT33A(encodes keratin 33A) were two correlated genes from black module, ADIPOQ (encodes adiponectin) was from salmon module, the three genes were positively correlated with DMD age. KNL1(encodes kinetochore scaffold 1) and CEP55(encodes centrosomal protein 55) were two genes that negatively correlated with DMD age. It is reported that adiponectin regulates senescence in keratinocytes $[35,36]$. Therefore, KRT31, KRT33A and ADIPOQ genes may interact with each other in DMD patients with different age. KNL1 and CEP55 are two genes associated with cellular cycling, and therefore, they may correlate with DMD age. In addition, it is reported that the expression of some centrosomal proteins decreases in muscular dystrophy $[37,38]$.

COL5A1(encodes collagen type $\mathrm{V}$ alpha 1 chain) and ADAMTS2(encodes ADAM metallopeptidase with thrombospondin type 1 motif 2 ) were common hub genes for BMD and BMD age. The accumulation of collagens is the feature of skeletal muscle fibrosis in BMD patients [39], therefore, collagen associated genes are hub genes and positively correlated with BMD. In addition, it seems that the expression level of collagen including COL5A1 
Table 1 Hub genes or highly correlated genes for clinical traits

\begin{tabular}{|c|c|c|c|c|c|c|c|c|c|}
\hline Clinical traits & Gene symbol & Module & $\begin{array}{l}\text { Positive or } \\
\text { negative }\end{array}$ & Remarks & $\begin{array}{l}\text { Clinical } \\
\text { traits }\end{array}$ & $\begin{array}{l}\text { Gene } \\
\text { symbol }\end{array}$ & Module & $\begin{array}{l}\text { Positive or } \\
\text { negative }\end{array}$ & Remarks \\
\hline \multirow[t]{10}{*}{ DMD } & TYROBP & Yellow & Positive & Hub gene & BMD & LOX & Blue & Positive & Hub gene \\
\hline & C3AR1 & Turquoise & Positive & Hub gene & & ELN & Blue & Positive & Hub gene \\
\hline & PLEK & Yellow & Positive & Hub gene & & PLEK & Blue & Positive & Hub gene \\
\hline & TLR7 & Turquoise & Positive & Hub gene & & BCL2L1 & Turquoise & Negative & Hub gene \\
\hline & LAPTM5 & Brown & Positive & Hub gene & & IKZF1 & Blue & Positive & Hub gene \\
\hline & AlF1 & Yellow & Positive & Hub gene & & CTSK & Blue & Positive & Hub gene \\
\hline & IRF8 & Turquoise & Positive & Hub gene & & THBS2 & Blue & Positive & Hub gene \\
\hline & FYB & Turquoise & Positive & Hub gene & & ADAMTS2 & Blue & Positive & Hub gene \\
\hline & CD33 & Turquoise & Positive & Hub gene & & CDK2 & Turquoise & Negative & Hub gene \\
\hline & NT5E & $\operatorname{Tan}$ & Positive & Hub gene & & COL5A1 & Blue & Positive & Hub gene \\
\hline \multirow[t]{8}{*}{ DMD age } & KRT31 & Black & Positive & $\begin{array}{l}\text { Correlated } \\
\text { genes }\end{array}$ & BMD age & COL14A1 & Blue & Negative & Hub gene \\
\hline & KRT33A & Black & Positive & $\begin{array}{l}\text { Correlated } \\
\text { genes }\end{array}$ & & ECT2 & Yellow & Negative & Hub gene \\
\hline & KNL1 & Blue & Negative & $\begin{array}{l}\text { Correlated } \\
\text { genes }\end{array}$ & & PARPBP & Blue & Negative & Hub gene \\
\hline & CEP55 & Green yellow & Negative & $\begin{array}{l}\text { Correlated } \\
\text { genes }\end{array}$ & & COL5A1 & Blue & Negative & Hub gene \\
\hline & ADIPOQ & Salmon & Positive & $\begin{array}{l}\text { Correlated } \\
\text { genes }\end{array}$ & & ADAMTS2 & Blue & Negative & Hub gene \\
\hline & & & & & $\begin{array}{l}\text { BMD pathol- } \\
\text { ogy }\end{array}$ & RPS4Y1 & Turquoise & Positive & $\begin{array}{l}\text { Correlated } \\
\text { gene }\end{array}$ \\
\hline & & & & & & KDM5D & Turquoise & Positive & $\begin{array}{l}\text { Correlated } \\
\text { gene }\end{array}$ \\
\hline & & & & & & CXCL5 & Turquoise & Positive & $\begin{array}{l}\text { Correlated } \\
\text { gene }\end{array}$ \\
\hline
\end{tabular}

and COL14A1 genes is negatively related to BMD age, a previous study suggested that the collagen level should increase in dystrophic mice [16]. However, we speculated that the possible reason for negatively relationship between collagen gene and BMD age may result from the relatively light symptom in the aging patients with BMD, and the muscle damage is also light, thus causing low collagen expression level compared with younger and severe patients. ADAMTS2 gene is involved in collagen processing [40]. In addition, it is reported that ADAMTS2 gene is also involved in aging [41].

Eight hub genes for BMD were from blue module, and all of them were positively correlated with BMD, these genes included LOX (encodes lysyl oxidase), ELN (encodes elastin), PLEK, IKZF1(encodes IKAROS family zinc finger 1), CTSK (encodes cathepsin K), THBS2(encodes thrombospondin 2), ADAMTS2 and COL5A1. LOX gene is involved in fibrogenesis, as well as collagen and elastin cross-linking, it is observed that its expression level is increased in mice and dogs with muscular dystrophy [42, 43], therefore, LOX, as well as ELN gene may be positively correlated with patients with BMD. It has been observed that overexpression of IKZF1 gene can upregulate matrix metalloproteinase, which plays an important role in BMD [44]. Collagens are major constituents of the extracellular matrix (ECM), while Cathepsin K plays an important role in ECM degradation [45]. Thrombospondin 2 can regulate the production of ECM and LOX protein levels [46]. PARPBP (encodes PARP1 binding protein) is another hub gene that from blue module, it is negatively related to BMD age. It is also associated with ECM [47]. Studies have demonstrated its role in BMD $[48,49]$. The genes in blue modules are all involved in ECM, which suggests the vital role of ECM in $\mathrm{BMD}$ and BMD age, this is consistent with previous studies $[50,51]$.

BCL2L1(encodes BCL2 like 1) and CDK2(encodes cyclin dependent kinase 2) were two hub genes from turquoise module and were both negatively correlated with $\mathrm{BMD}$. It has been observed that BCL2L1 protein is a part of signaling pathway that can promote cell division [52]. CDK2 protein participates in cell cycling, the negatively relationship between the two genes and BMD indicates a muscular damage and dystrophy [53]. RPS4Y1(encodes ribosomal protein S4, Y-linked 1), KDM5D(encodes lysine demethylase 5D) and CXCL5(encodes C-X-C motif 
Table 2 GO and KEGG pathway analysis that hub genes were enriched in

\begin{tabular}{|c|c|c|c|}
\hline Traits & Enrichment & Type & Gene symbol \\
\hline \multirow[t]{23}{*}{$\mathrm{DMD}$} & Immune response & $\mathrm{BP}$ & IRF8,CD33,C3AR1,TLR7,TYROBP,AIF1 \\
\hline & Myeloid leukocyte activation & $\mathrm{BP}$ & CD33,C3AR1,TLR7,TYROBP,AIF1 \\
\hline & Regulated exocytosis & $\mathrm{BP}$ & CD33,C3AR1,PLEK,TYROBP \\
\hline & Cell activation & $\mathrm{BP}$ & CD33,C3AR1,TLR7,PLEK,TYROBP,AIF1 \\
\hline & Neutrophil degranulation & $\mathrm{BP}$ & CD33,C3AR1,TYROBP \\
\hline & Neutrophil activation involved in immune response & $\mathrm{BP}$ & CD33,C3AR1,TYROBP \\
\hline & Defense response & $\mathrm{BP}$ & IRF8,C3AR1,TLR7,TYROBP,AIF1 \\
\hline & Neutrophil activation & $\mathrm{BP}$ & CD33,C3AR1,TYROBP \\
\hline & Neutrophil mediated immunity & $\mathrm{BP}$ & CD33,C3AR1,TYROBP \\
\hline & Granulocyte activation & $\mathrm{BP}$ & CD33,C3AR1,TYROBP \\
\hline & Secretory vesicle & CC & CD33,C3AR1,TYROBP \\
\hline & Secretory granule & CC & CD33,C3AR1,TYROBP \\
\hline & Lysosome & $\mathrm{CC}$ & C3AR1,TLR7 \\
\hline & Lytic vacuole & $\mathrm{CC}$ & C3AR1,TLR7 \\
\hline & Vacuolar part & $\mathrm{CC}$ & C3AR1,TLR7 \\
\hline & Cytoplasmic vesicle part & CC & CD33,C3AR1,TLR7,TYROBP \\
\hline & Vacuole & $\mathrm{CC}$ & C3AR1,TLR7 \\
\hline & Ruffle & CC & PLEK;AIF1 \\
\hline & Whole membrane & CC & CD33,C3AR1,TLR7 \\
\hline & Protein-containing complex binding & MF & AlF1 \\
\hline & Lipid binding & MF & PLEK \\
\hline & Staphylococcus aureus infection & KEGG & C3AR1 \\
\hline & Pertussis & KEGG & IRF8 \\
\hline \multirow[t]{23}{*}{ BMD } & Collagen fibril organization & $\mathrm{BP}$ & COL5A1,LOX,ADAMTS2 \\
\hline & Extracellular matrix organization & BP & CTSK,COL5A1,ELN,LOX \\
\hline & Positive regulation of phosphatase activity & $\mathrm{BP}$ & PLEK \\
\hline & Positive regulation of integrin activation & $\mathrm{BP}$ & PLEK \\
\hline & Extracellular structure organization & $\mathrm{BP}$ & CTSK,COL5A1,ELN,LOX,ADAMTS2 \\
\hline & Cell adhesion & $\mathrm{BP}$ & THBS2;PLEK,COL5A1 \\
\hline & Biological adhesion & $\mathrm{BP}$ & THBS2,PLEK,COL5A1 \\
\hline & Extracellular matrix & CC & THBS2,COL5A1,ELN,LOX,ADAMTS2 \\
\hline & Collagen-containing extracellular matrix & CC & THBS2,COL5A1,ELN,ADAMTS2 \\
\hline & Basement membrane & CC & THBS2,COL5A1 \\
\hline & Extracellular matrix component & $\mathrm{CC}$ & COL5A1 \\
\hline & Cell projection part & $\mathrm{CC}$ & PLEK \\
\hline & Plasma membrane bounded cell projection part & $\mathrm{CC}$ & PLEK \\
\hline & Extracellular matrix structural constituent & MF & COL5A1,ELN,THBS2 \\
\hline & Structural molecule activity & MF & COL5A1,ELN,THBS2 \\
\hline & Protein kinase $\mathrm{C}$ binding & MF & PLEK \\
\hline & Magnesium ion binding & MF & CDK2 \\
\hline & Fibronectin binding & MF & CTSK \\
\hline & p53 signaling pathway & KEGG & CDK2,BCL2L1 \\
\hline & Protein digestion and absorption & KEGG & COL5A1,ELN \\
\hline & Rheumatoid arthritis & KEGG & CTSK \\
\hline & Amyotrophic lateral sclerosis (ALS) & KEGG & $\mathrm{BCL} 2 \mathrm{~L} 1$ \\
\hline & PI3K-Akt signaling pathway & KEGG & THBS2,CDK2,BCL2L1 \\
\hline
\end{tabular}


Table 2 (continued)

\begin{tabular}{llll}
\hline Traits & Enrichment & Type & Gene symbol \\
\hline BMD age & Collagen fibril organization & BP & COL5A1,COL14A1,ADAMTS2 \\
& Endodermal cell differentiation & BP & COL5A1 \\
Extracellular matrix organization & BP & COL5A1,COL14A1,ADAMTS2 \\
Endoderm formation & BP & COL5A1 \\
Extracellular structure organization & BP & COL5A1,COL14A1,ADAMTS2 \\
Endoderm development & BP & COL5A1 \\
Extracellular matrix & CC & COL5A1,COL14A1,ADAMTS2 \\
Collagen-containing extracellular matrix & CC ADAMTS2 \\
Extracellular matrix component & CC & COL5A1,COL14A1 \\
Extracellular matrix structural constituent & MF & COL5A1,COL14A1 \\
DNA binding, bending & MF & COL5A1,COL14A1 \\
Structural molecule activity & MF & COL5A1,COL14A1 \\
Extracellular matrix structural constituent conferring tensile & MF & COL5A1,COL14A1
\end{tabular}

chemokine ligand 5) were also from turquoise module and all positively correlated with BMD pathology. RPS4Y1 and KDM5D genes are both from Y chromosome. It has been demonstrated that they both participate in cell cycle, which suggests that turquoise module mainly involves in cell cycle [54-56]. ECT2(encodes epithelial cell transforming 2) was from yellow module, it was a hub gene and negatively correlated with BMD age. It is reported that ECT2 gene is an oncogene and associated with senescence [57].

The GO and KEGG pathway analysis of all hub genes for DMD, BMD and age indicated that the enrichment mainly involves immune and inflammation for DMD, while hub genes for BMD mainly enriched in ECM, this is consistent with our analysis of hub gene. This indicated that DMD and BMD may differ in the pathological mechanism, the different pathological mechanisms between the two diseases may provide new pharmaceutical therapy for DMD and BMD. Compared with the previous bioinformatic study using the same dataset [12], only a few hub genes were the same between the two papers, this may lie in the application of whole gene array in their study and DEGs in our study. However, both studies have found the immune system may be involved in DMD, this suggests the its potential key role in DMD.

There still exist several limitations that may influence our results. Firstly, the number of genes in each clustered module was small, and the number of genes in the non-clustered grey module was large, which forced us to analyse all correlated clustered genes instead of only one module. Secondly, the number of correlated clustered genes in DMD age and BMD pathology was too small to conduct enrichment and PPI analysis, in addition, all correlated genes for DMD pathology were from grey module. Thirdly, basic demographics characteristics (such as gender and age) of healthy individuals were not applicable, and the number of the three groups was also not big enough. Lastly, the difference between the selected threshold and the real line in Fig. 3 was larger than was ideal.

\section{Conclusion}

In conclusion, several hub genes are identified for DMD: C3AR1, TLR7, IRF8, FYB and CD33(immune and inflammation associated genes), TYROBP, PLEK, AIF1(actin reorganization associated genes), LAPTM5 and NT5E(cell death and arterial calcification associated genes, respectively). In BMD, a number of hub genes are identified: LOX, ELN, PLEK, IKZF1, CTSK, THBS2, ADAMTS2, COL5A1(ECM associated genes), BCL2L1 and CDK2(cell cycle associated genes). Keratin may play an important role in DMD age, while ECM may play a key role in BMD age, and cell cycle may be associated with BMD pathology. It is important to diagnose and treat DMD and BMD at an early age via the expression level of hub genes. Further studies are required to explore the relevant genes in DMD and BMD, as well as pharmaceutical therapies aimed at these targets. 


\section{Abbreviations}

BMD: Becker muscular dystrophy; DGC: Dystrophin-glycoprotein complex; DMD: Duchenne muscular dystrophy; FC: Fold change; FDR: False discovery rate; GEO: Gene Expression Omnibus; GO: Gene Ontology; KEGG: Kyoto Encyclopaedia of Genes and Genomes; PPI: Protein-protein interaction; STRING: Search Tool for the Retrieval of Interacting Genes; TOM: Topological overlap matrix; WGCNA: Weighted correlation network analysis.

\section{Acknowledgements}

The study was sponsored by the Shandong Natural Science Foundation (ZR2019MH097).

\section{Authors' contributions}

Junjie Wang: designed research; conducted analysis; wrote paper; checked paper. Qin Fan: conducted analysis; checked paper. Tengbo Yu: designed research; checked paper. Yingze Zhang: designed research; checked paper. The author(s) read and approved the final manuscript.

\section{Funding}

None.

\section{Availability of data and materials}

As a bioinformatics analysis, there are no patient data sets.

\section{Declarations}

\section{Ethics approval and consent to participate}

Not applicable.

\section{Consent for publication}

Not applicable.

\section{Competing interests}

The authors declare that they have no competing interests.

\section{Author details}

${ }^{1}$ Qingdao University, No.308, Ningxia Road, Qingdao, Shandong Province 266000, China. ${ }^{2}$ Orthopaedic Center, The Affiliated Hospital of Qingdao University, No. 16, Jiangsu Road, Qingdao, Shandong Province 266000, China. ${ }^{3}$ Department of Orthopaedic Surgery, Third Hospital of Hebei Medical University, No. 139, Ziqiang Road, Shijiazhuang, Hebei Province 050000, China.

Received: 28 July 2021 Accepted: 19 November 2021

Published online: 18 December 2021

\section{References}

1. Birnkrant DJ, Bushby K, Bann CM, Apkon SD, Blackwell A, Brumbaugh D, et al. Diagnosis and management of Duchenne muscular dystrophy, part 1: diagnosis, and neuromuscular, rehabilitation, endocrine, and gastrointestinal and nutritional management. Lancet Neurol. 2018;17(3):251-67.

2. Blake DJ, Weir A, Newey SE, Davies KE. Function and genetics of dystrophin and dystrophin-related proteins in muscle. Physiol Rev. 2002;82(2):291-329.

3. Haslett JN, Sanoudou D, Kho AT, Bennett RR, Greenberg SA, Kohane IS, et al. Gene expression comparison of biopsies from Duchenne muscular dystrophy (DMD) and normal skeletal muscle. Proc Natl Acad Sci U S A. 2002;99(23):15000-5.

4. Emery AE. The muscular dystrophies. Lancet (London, England). 2002;359(9307):687-95.

5. Waldrop MA, Flanigan KM. Update in Duchenne and Becker muscular dystrophy. Curr Opin Neurol. 2019;32(5):722-7.

6. Ferreiro V, Giliberto F, Muñiz GM, Francipane L, Marzese DM, Mampel A, et al. Asymptomatic Becker muscular dystrophy in a family with a multiexon deletion. Muscle Nerve. 2009;39(2):239-43.

7. Mendell JR, Shilling C, Leslie ND, Flanigan KM, al-Dahhak R, Gastier-Foster J, et al. Evidence-based path to newborn screening for Duchenne muscular dystrophy. Ann Neurol. 2012;71(3):304-13.
8. Mah JK, Korngut L, Dykeman J, Day L, Pringsheim T, Jette N. A systematic review and meta-analysis on the epidemiology of Duchenne and Becker muscular dystrophy. Neuromuscul Disord. 2014;24(6):482-91.

9. Wong BL, Rybalsky I, Shellenbarger KC, Tian C, McMahon MA, Rutter MM, et al. Long-term outcome of interdisciplinary management of patients with Duchenne muscular dystrophy receiving daily glucocorticoid treatment. J Pediatr. 2017;182:296-303.e1.

10. Eagle M, Baudouin SV, Chandler C, Giddings DR, Bullock R, Bushby K. Survival in Duchenne muscular dystrophy: improvements in life expectancy since 1967 and the impact of home nocturnal ventilation. Neuromuscul Disord. 2002;12(10):926-9.

11. Straub V, Balabanov P, Bushby K, Ensini M, Goemans N, De Luca A, et al. Stakeholder cooperation to overcome challenges in orphan medicine development: the example of Duchenne muscular dystrophy. Lancet Neurol. 2016;15(8):882-90.

12. Xu X, Hao Y, Wu J, Zhao J, Xiong S. Assessment of weighted gene coexpression network analysis to explore key pathways and novel biomarkers in muscular dystrophy. Pharmacogenomics Pers Med. 2021;14:431-44.

13. Seldeen KL, Thiyagarajan R, Redae Y, Jacob A, Troen BR, Quigg RJ, et al. Absence of complement factor $\mathrm{H}$ reduces physical performance in C57BL6 mice. Immunobiology. 2020;225(5):152003.

14. Puri TS, Quigg RJ. The many effects of complement C3- and C5-binding proteins in renal injury. Semin Nephrol. 2007;27(3):321-37.

15. Han R, Frett EM, Levy JR, Rader EP, Lueck JD, Bansal D, et al. Genetic ablation of complement C3 attenuates muscle pathology in dysferlindeficient mice. J Clin Invest. 2010;120(12):4366-74.

16. Colussi C, Banfi C, Brioschi M, Tremoli E, Straino S, Spallotta F, et al. Proteomic profile of differentially expressed plasma proteins from dystrophic mice and following suberoylanilide hydroxamic acid treatment. Proteomics Clin Appl. 2010;4(1):71-83.

17. Henriques-Pons A, Yu Q, Rayavarapu S, Cohen TV, Ampong B, Cha HJ, et al. Role of toll-like receptors in the pathogenesis of dystrophin-deficient skeletal and heart muscle. Hum Mol Genet. 2014;23(10):2604-17.

18. Chmielewski S, Piaszyk-Borychowska A, Wesoly J, Bluyssen HA. STAT1 and IRF8 in vascular inflammation and cardiovascular disease: diagnostic and therapeutic potential. Int Rev Immunol. 2016:35(5):434-54.

19. Jeong H, Shin JY, Lee K, Lee SJ, Chong HJ, Jeong H, et al. Caffeoyl-prolylhistidine amide inhibits fyn and alleviates atopic dermatitis-like phenotypes via suppression of NF-kB activation. Int J Mol Sci. 2020;21(19):7160.

20. Paranjape A, Haque TT, Kiwanuka KN, Qayum AA, Barnstein BO, Finkelman FD, et al. The Fyn-Stat5 cascade is required for Fcy receptor-mediated mast cell function. Cell Immunol. 2020:356:104134.

21. Crocker PR, McMillan SJ, Richards HE. CD33-related siglecs as potential modulators of inflammatory responses. Ann N Y Acad Sci. 2012;1253:102-11.

22. Zhang LX, Ye J, Chen YB, Peng HL, Chen $X$, Liu L, et al. The effect of CD33 expression on inflammatory response in chronic obstructive pulmonary disease. Immunol Investig. 2013;42(8):701-10.

23. Rosenberg AS, Puig M, Nagaraju K, Hoffman EP, Villalta SA, Rao VA, et al. Immune-mediated pathology in Duchenne muscular dystrophy. Sci Transl Med. 2015;7(299):299rv4.

24. Messina S, Bitto A, Aguennouz M, Mazzeo A, Migliorato A, Polito F, et al. Flavocoxid counteracts muscle necrosis and improves functional properties in mdx mice: a comparison study with methylprednisolone. Exp Neurol. 2009;220(2):349-58.

25. Hamaguchi N, Ihara S, Ohdaira T, Nagano H, Iwamatsu A, Tachikawa H, et al. Pleckstrin-2 selectively interacts with phosphatidylinositol 3-kinase lipid products and regulates actin organization and cell spreading. Biochem Biophys Res Commun. 2007;361(2):270-5.

26. Polanowska-Grabowska R, Gear AR. Activation of protein kinase $C$ is required for the stable attachment of adherent platelets to collagen but is not needed for the initial rapid adhesion under flow conditions. Arterioscler Thromb Vasc Biol. 1999;19(12):3044-54.

27. Vicente-Manzanares M, Sánchez-Madrid F. Role of the cytoskeleton during leukocyte responses. Nat Rev Immunol. 2004;4(2):110-22.

28. Autieri MV, Kelemen SE, Wendt KW. AIF-1 is an actin-polymerizing and Rac1-activating protein that promotes vascular smooth muscle cell migration. Circ Res. 2003;92(10):1107-14.

29. Köhler C. Allograft inflammatory factor-1/ionized calciumbinding adapter molecule 1 is specifically expressed by most 
subpopulations of macrophages and spermatids in testis. Cell Tissue Res. 2007;330(2):291-302.

30. Thompson TG, Chan YM, Hack AA, Brosius M, Rajala M, Lidov HG, et al. Filamin 2 (FLN2): a muscle-specific sarcoglycan interacting protein. J Cell Biol. 2000;148(1):115-26.

31. Inoue J, Misawa A, Tanaka Y, Ichinose S, Sugino Y, Hosoi H, et al. Lysosomal-associated protein multispanning transmembrane 5 gene (LAPTM5) is associated with spontaneous regression of neuroblastomas. PLoS One. 2009:4(9):e7099.

32. Durnin L, Kurahashi M, Sanders KM. Extracellular metabolism of the enteric inhibitory neurotransmitter $\beta$-nicotinamide adenine dinucleotide ( $\beta$-NAD) in the murine colon. J Physiol. 2020;598(20):4509-21.

33. Hofbauer LC, Hamann C, Schoppet M. NT5E mutations and arterial calcifications. N Engl J Med. 2011;364(16):1578 author reply 9-80.

34. Ryan TD, Parent JJ, Gao Z, Khoury PR, Dupont E, Smith JN, et al. Central arterial function measured by non-invasive pulse wave analysis is abnormal in patients with Duchenne muscular dystrophy. Pediatr Cardiol. 2017;38(6):1269-76.

35. Jin T, Kim MJ, Heo WI, Park KY, Choi SY, Lee MK, et al. Adiponectin corrects premature cellular senescence and normalizes antimicrobial peptide levels in senescent keratinocytes. Biochem Biophys Res Commun. 2016:477(4):678-84.

36. Kim M, Park KY, Lee MK, Jin T, Seo SJ. Adiponectin suppresses UVBinduced premature senescence and $\mathrm{hBD} 2$ overexpression in human keratinocytes. PLoS One. 2016;11(8):e0161247.

37. Gimpel P, Lee YL, Sobota RM, Calvi A, Koullourou V, Patel R, et al. Nesprin1a-dependent microtubule nucleation from the nuclear envelope via Akap450 is necessary for nuclear positioning in muscle cells. Curr Biol. 2017;27(19):2999-3009.e9.

38. Meinke P, Mattioli E, Haque F, Antoku S, Columbaro M, Straatman KR, et al. Muscular dystrophy-associated SUN1 and SUN2 variants disrupt nuclearcytoskeletal connections and myonuclear organization. PLoS Genet. 2014;10(9):e1004605.

39. Regensburger AP, Fonteyne LM, Jüngert J, Wagner AL, Gerhalter T. Detection of collagens by multispectral optoacoustic tomography as an imaging biomarker for Duchenne muscular dystrophy. Nat Med. 2019;25(12):1905-15.

40. Colige A, Nuytinck L, Hausser I, van Essen AJ, Thiry M, Herens C, et al. Novel types of mutation responsible for the dermatosparactic type of Ehlers-Danlos syndrome (type $\mathrm{VIIC}$ ) and common polymorphisms in the ADAMTS2 gene. J Investig Dermatol. 2004;123(4):656-63.

41. Soundararajan R, Stearns TM, Czachor A, Fukumoto J, Turn C, Westermann-Clark E, et al. Global gene profiling of aging lungs in Atp8b1 mutant mice. Aging. 2016;8(9):2232-52.

42. Desguerre I, Arnold L, Vignaud A, Cuvellier S, Yacoub-Youssef H, Gherardi RK, et al. A new model of experimental fibrosis in hindlimb skeletal muscle of adult mdx mouse mimicking muscular dystrophy. Muscle Nerve. 2012;45(6):803-14.

43. Smith LR, Hammers DW, Sweeney HL, Barton ER. Increased collagen cross-linking is a signature of dystrophin-deficient muscle. Muscle Nerve. 2016;54(1):71-8.

44. Hung JJ, Kao YS, Huang CH, Hsu WH. Overexpression of Aiolos promotes epithelial-mesenchymal transition and cancer stem cell-like properties in lung cancer cells. Sci Rep. 2019;9(1):2991.

45. Gruber HE, Ingram JA, Hoelscher GL, Zinchenko N, Norton HJ, Hanley EN $\mathrm{Jr}$. Constitutive expression of cathepsin $\mathrm{K}$ in the human intervertebral disc: new insight into disc extracellular matrix remodeling via cathepsin $\mathrm{K}$ and receptor activator of nuclear factor-KB ligand. Arthritis Res Ther. 2011;13(4):R140

46. Calabro NE, Barrett A, Chamorro-Jorganes A, Tam S, Kristofik NJ, Xing $\mathrm{H}$, et al. Thrombospondin-2 regulates extracellular matrix production, LOX levels, and cross-linking via downregulation of miR-29. Matrix Biol. 2019;82:71-85.

47. Ke J, Liu F, Tu Y, Cai Z, Luo Y, Wu X. PARP1-RNA interaction analysis: PARP1 regulates the expression of extracellular matrix-related genes in HK-2 renal proximal tubular epithelial cells. FEBS Lett. 2021;595(9):1375-87.

48. Yu S, Wang X, Geng P, Tang X, Xiang L, Lu X, et al. Melatonin regulates PARP1 to control the senescence-associated secretory phenotype (SASP) in human fetal lung fibroblast cells. J Pineal Res. 2017;63(1). https://doi. org/10.1111/jpi.12405.
49. Mendelsohn AR, Larrick JW. The NAD+/PARP1/SIRT1 Axis in aging. Rejuvenation Res. 2017;20(3):244-7.

50. Capitanio D, Moriggi M, Torretta E, Barbacini P, De Palma S, Viganò A, et al. Comparative proteomic analyses of Duchenne muscular dystrophy and Becker muscular dystrophy muscles: changes contributing to preserve muscle function in Becker muscular dystrophy patients. J Cachexia Sarcopenia Muscle. 2020;11(2):547-63.

51. van den Bergen JC, Wokke BH, Hulsker MA, Verschuuren JJ, Aartsma-Rus AM. Studying the role of dystrophin-associated proteins in influencing Becker muscular dystrophy disease severity. Neuromuscul Disord. 2015;25(3):231-7.

52. Yang Z, An Y, Wang N, Dong X, Kang H. LINC02595 promotes tumor progression in colorectal cancer by inhibiting miR-203b-3p activity and facilitating BCL2L1 expression. J Cell Physiol. 2020;235(10):7449-64

53. Ortega S, Prieto I, Odajima J, Martín A, Dubus P, Sotillo R, et al. Cyclindependent kinase 2 is essential for meiosis but not for mitotic cell division in mice. Nat Genet. 2003;35(1):25-31.

54. Cui D, Zhao Y, Xu J. Activation of CXCL5-CXCR2 axis promotes proliferation and accelerates $\mathrm{G} 1$ to $S$ phase transition of papillary thyroid carcinoma cells and activates JNK and p38 pathways. Cancer Biol Ther. 2019;20(5):608-16.

55. Jangravi Z, Tabar MS, Mirzaei M, Parsamatin P, Vakilian H, Alikhani M, et al. Two splice variants of $Y$ chromosome-located lysine-specific demethylase 5D have distinct function in prostate cancer cell line (DU-145). J Proteome Res. 2015;14(9):3492-502.

56. Chen $X$, Tong $C, L i H$, Peng W, Li R, Luo X, et al. Dysregulated expression of RPS4Y1 (ribosomal protein S4, Y-linked 1) impairs STAT3 (signal transducer and activator of transcription 3) signaling to suppress trophoblast cell migration and invasion in preeclampsia. Hypertension (Dallas, Tex: 1979). 2018;71(3):481-90.

57. Cairney CJ, Godwin LS, Bilsland AE, Burns S. A 'synthetic-sickness' screen for senescence re-engagement targets in mutant cancer backgrounds. PLoS Genet. 2017;13(8):e1006942.

\section{Publisher's Note}

Springer Nature remains neutral with regard to jurisdictional claims in published maps and institutional affiliations.

Ready to submit your research? Choose BMC and benefit from:

- fast, convenient online submission

- thorough peer review by experienced researchers in your field

- rapid publication on acceptance

- support for research data, including large and complex data types

- gold Open Access which fosters wider collaboration and increased citations

- maximum visibility for your research: over $100 \mathrm{M}$ website views per year

At BMC, research is always in progress.

Learn more biomedcentral.com/submissions 